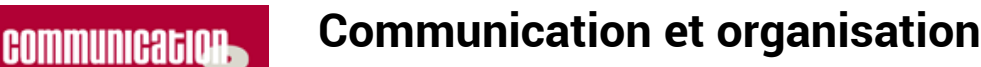

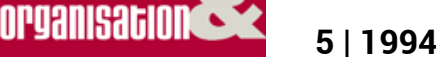

La communication interne : une approche croisée

\title{
La communication interne : une définition en évolution
}

Nicole Giroux

\section{OpenEdition}

\section{Journals}

Édition électronique

URL : http://journals.openedition.org/communicationorganisation/1696

DOI : 10.4000/communicationorganisation. 1696

ISSN : $1775-3546$

Éditeur

Presses universitaires de Bordeaux

Édition imprimée

Date de publication : 1 mai 1994

ISSN : 1168-5549

Référence électronique

Nicole Giroux, «La communication interne : une définition en évolution », Communication et organisation [En ligne], 5 | 1994, mis en ligne le 26 mars 2012, consulté le 10 décembre 2020. URL

http://journals.openedition.org/communicationorganisation/1696; DOI : https://doi.org/10.4000/ communicationorganisation. 1696

Ce document a été généré automatiquement le 10 décembre 2020.

(c) Presses universitaires de Bordeaux 


\title{
La communication interne: une définition en évolution
}

\author{
Nicole Giroux
}

1 Nous remercions Christiane Demers, Richard Déry, Luc Giroux, Pierre Mévellec et James Taylor pour leurs judicieux commentaires sur les versions préliminaires de cet article.

\section{Introduction}

2 L'intérêt pour la communication organisationnelle comme champ d'étude à part entière est relativement récent. En effet, Redding (1985) situe son émergence à la fin des années 40. Sa courte histoire s'inscrit donc toute entière à l'intérieur de la durée d'une vie humaine. Ce nouveau champ se situe à l'interface des sciences de la communication et de celles de l'organisation. Il s'est développé en s'appuyant sur les modèles issus de ces deux disciplines. Avec le temps, on en est venu à distinguer deux dimensions de la communication organisationnelle : la communication externe qui relie l'entreprise et ses partenaires dans l'environnement, la communication interne entre les membres de l'organisation. Au cours des dernières années, la communication interne a attiré l'attention des chercheurs en gestion qui y voient la clé du renouvellement de l'organisation (Bartoli, 1990 ; Auvinet et al. 1990)

3 Les chercheurs et les praticiens qui s'intéressent à la communication interne situent leurs réflexions ou leurs interventions dans le cadre d'une définition implicite ou, plus rarement, explicite de celle-ci. Puisque le choix d'une définition peut avoir des conséquences importantes sur la démarche des chercheurs ou sur les stratégies d'intervention des praticiens, il nous apparaît pertinent de bien comprendre toutes les implications de la définition retenue.

4 Dans ce texte, nous présenterons des définitions de la communication interne qui sont apparues successivement au fil de l'évolution des modèles de l'organisation et des modèles de la communication. Ces définitions relèvent de deux courants de pensée : la perspective fonctionnaliste et la perspective interprétative. Dans le courant 
fonctionnaliste on retrouve deux définitions: la communication productive et la communication intégratrice. Dans le courant interprétatif, la communication est définie comme «organisante». Ces trois définitions ont été retenues parce qu'elles nous semblent capturer l'essence de la fonction de la communication dans les organisations à savoir la création, le maintien et le renouvellement d'une collectivité productive et autoproductrice.

Chacune de ces définitions de la communication interne repose sur des conceptions de la communication et de l'organisation qui sont sensiblement différentes. Ces définitions réfèrent à des conceptualisations différentes de la structure, de la culture et du pouvoir dans l'organisation. Elles mettent l'emphase sur des aspects différents de la communication, proposent à celle-ci des buts distincts et soulèvent des problèmes qui leur sont particuliers (voir Tableau 1).

6 Ces définitions constituent des points de vue alternatifs qui éclairent de façon privilégiée certains aspects de la communication interne. C'est pourquoi, dans ce texte, nous décrirons dans un premier temps les perspectives fonctionnaliste et interprétative et les définitions qu'elles proposent de la communication interne en mettant l'accent sur leurs différences et, dans un second temps, nous montrerons comment la communication « organisante » incorpore et transforme les acquis des deux définitions précédentes.

\section{La perspective fonctionnaliste}

7 La perspective fonctionnaliste, qui est issue de la sociologie de la régulation (Burrel et Morgan, 1979), observe la société et l'organisation d'un point de vue objectiviste. Elle s'attache à expliquer l'ordre, le consensus, l'intégration dans les grands ensembles organisés. Cette perspective met l'accent sur la rationalité et le pragmatisme. Elle vise à comprendre le fonctionnement des systèmes et à prescrire au besoin des méthodes visant à en améliorer la performance. La perspective fonctionnaliste est la plus ancienne et elle est encore dominante aujourd'hui dans les champs de la gestion et de la communication.

\section{La communication productive}

8 La communication productive c'est la communication vue comme un outil de production. Cette définition de la communication présente celle-ci comme un message transmis par un émetteur à récepteur à travers des canaux. Cette représentation classique de la communication repose sur une vision mécaniste. Elle correspond, en théorie de l'organisation, à la représentation de l'organisation comme machine que l'on retrouve chez les auteurs classiques comme Taylor, Fayol et Weber. Elle a aussi son pendant dans une vision mécaniste plus sophistiquée qui compare l'organisation à un cerveau ou à un ordinateur ou encore à une configuration, vision présente dans les travaux d'auteurs néoclassiques comme Simon et Mintzberg (Aktouf, 1989a ; Morgan, 1989).

Cette vision instrumentale de la communication suppose que l'émetteur fait quelque chose au receveur à travers le message qu'il envoie (Krone et al., 1987). Elle met l'accent sur le message qui est en quelque sorte le réceptacle de la signification créée par l'émetteur. L'émetteur agit sur le récepteur en transmettant la signification qui est présentée comme une réalité objective qu'il suffit de décoder. 
10 Cette définition de la communication concorde avec la vision mécaniste de l'organisation qui la conçoit comme un tout, une entité productive, hiérarchisée dont les membres, conçus comme de simples rouages, sont motivés par une rétribution avant tout économique. L'organisation est alors divisée entre ceux qui savent, les gestionnaires, et ceux qui réalisent, les travailleurs; entre ceux qui émettent et ceux qui reçoivent les messages. La division du travail vient alors consacrer la répartition du pouvoir dans l'organisation: le pouvoir légitime est entre les mains de la direction qui délègue des responsabilités à certains membres de l'organisation.

11 La structure de l'organisation est formelle, c'est le plan du réseau de communication, la représentation des lignes d'autorité et de pouvoir. Dans cette vision traditionnelle de l'organisation, l'émetteur privilégié est le supérieur qui transmet à travers la hiérarchie ses directives à ses subordonnés. La question importante devient alors de déterminer l'étendue du contrôle du gestionnaire c'est-à-dire le nombre de subordonnés qu'il peut superviser, avec lesquels il peut communiquer efficacement.

12 Le contenu du message est avant tout opératoire (Laramée, 1989). Il est orienté vers le fonctionnement quotidien de l'organisation : il décrit la tâche à accomplir, les méthodes à utiliser, les résultats attendus. Il n'est pas ici question de sentiments ou de valeurs partagées. La rationalité de l'organisation est celle de la direction qui est seule créatrice de sens, les employés se soumettent aux directives parce que leur intérêt, en principe, est en concordance avec celui de la direction. Pour les auteurs classiques, qui prenaient pour modèles des institutions comme l'armée ou l'Église, l'organisation devait promouvoir les valeurs de la société de l'époque : ordre, stabilité, unité de direction.

Cette représentation de la communication interne tient pour acquis que le récepteur reçoit le message tel qu'il a été envoyé et que ce message va automatiquement contrôler les actions des travailleurs (Mc Daniel Johnson, 1977). C'est ce que Axley (1984) appelle la métaphore du conduit. Dans ce contexte, l'utilité de la communication repose sur sa capacité à faire réaliser par les récepteurs-subordonnés les objectifs de production souhaités par l'émetteur dirigeant. Il s'agit d'une communication linéaire, descendante et limitée à des fins productives (Sfez, 1991). Cette communication s'actualise dans un registre étroit et spécialisé où l'interactivité entre les membres de l'organisation est restreinte, certains membres de l'organisation étant même parfois condamnés au silence (Aktouf, 1989b ; Henriet et Boneu, 1990).

La communication productive est soumise au calcul économique. Il faut alors trouver la meilleure façon de produire le résultat escompté en minimisant les coûts associés à la communication. C'est pourquoi la communication y est surtout formelle, standardisée, écrite (Euske et Roberts, 1987 ; Rogers et Agarwala-Rogers, 1980). On peut l'observer sous sa forme émergente dans les directives données par un contremaître ou dans les mémos adressés par un chef de service. Dans sa forme planifiée, elle est cahier de règles et procédures administratives ou encore manuel destiné aux opérateurs.

comme, dans cette définition, la communication est vue comme un processus d'instruction qui informe les employés sur leurs responsabilités formelles et qui leur transmet les programmes d'action nécessaires à la production de biens et de services. Elle sert à diriger, coordonner et réguler les activités des membres de l'organisation (Farace et al., 1977).

16 Cette fonction de régulation et de coordination se retrouve aussi dans les descriptions plus modernes de l'organisation. Ainsi, dans sa description des différentes configurations 
organisationnelles, Mintzberg (1979) décrit six modes de coordination qui réfèrent à des formes particulières de communication productive. Ce sont : 1) la communication directe descendante que l'on observe dans la supervision directe au sein des entreprises simples ; 2) la communication écrite et descendante de la standardisation des procédés dans les bureaucraties mécanistes; 3) l'acquisition d'un langage technique commun et la délibération collégiale de la standardisation des qualifications dans les bureaucraties professionnelles ; 4) la communication descendante des objectifs et le contrôle de leur atteinte qui sont inclus dans la supervision des résultats dans les entreprises divisionnalisées ; 5) la communication horizontale, de face-à-face entre spécialistes qui réalisent l'ajustement mutuel dans les adhocraties; 6) la communication doctrinaire servant à la standardisation des normes dans les organisations missionnaires. Toutes ces formes de communication ont un même objectif : la réalisation de la production. Leur différence provient des particularités de la tâche à accomplir (simplicité/complexité, répétitive/non répétitive, requérant ou non l'autonomie des opérateurs) et des caractéristiques de l'environnement (simple/complexe, stable/dynamique).

Mais l'organisation ne produit pas que des biens et des services. Elle peut aussi être vue comme un système où se prennent, à tous les niveaux de la hiérarchie des décisions d'allocations et d'utilisation de ressources. En ce cas, elle produit aussi des informations pour la prise de décision. Elle en produirait même plus qu'il n'est nécessaire (Feldman et March, 1981).

18 Les auteurs de l'approche de la décision et de l'approche systémique, contrairement aux auteurs classiques qui faisaient peu de cas de la communication, ont collaboré davantage sur la question, venant enrichir et nuancer la description de la communication interne. Ils se sont intéressés à la communication comme processus de transfert de l'information. Pour eux, l'information est une réalité objective que l'on peut mesurer, faire circuler, emmagasiner et récupérer. Cette information constitue le matériau de base de la prise de décision. C'est pourquoi, ils ont souligné l'importance de la qualité de l'information et se sont préoccupés de l'efficacité du système de traitement de l'information. Ces auteurs n'ont pas tenu pour acquis la transparence et l'efficacité des messages mais ont plutôt souligné la présence de distorsion, de filtrage et de surcharge de l'information (Putnam, 1982).

19 Ils ont aussi, via la notion de rétroaction (« feedback»), introduit de la circularité dans le processus jusque là linéaire de communication. La communication n'est plus alors seulement un outil de direction et de contrôle social des personnes, elle devient aussi un moyen d'alimenter le système organisationnel en données pertinentes à l'élaboration de la décision. Elle est aussi un véhicule utile au contrôle des résultats. Il devient alors important de mettre en place des systèmes assurant la collecte, la circulation et le traitement de l'information. L'organisation devient un enchevêtrement de décisions, une cascade de programmes d'action, un réseau où circule l'information (March et Simon, 1958). La direction de la communication dans l'organisation, si elle demeure verticale, comme dans l'approche traditionnelle, se produit maintenant dans les deux sens. Le travailleur devient à son tour un émetteur (Farace et al., 1977). Il reçoit les informations nécessaires à la réalisation de sa tâche de production; il transmet aux gestionnaires les informations dont ceux-ci ont besoin pour prendre des décisions.

20 La définition de la communication-productive met l'emphase sur différents éléments de la communication : le message, les canaux et les réseaux de communication. Elle soulève différents problèmes tels que l'intelligibilité et la pertinence des messages. Ainsi, Simon 
(1945/1990) a fait ressortir l'importance d'adapter le message à l'auditoire tant au niveau sémantique qu'à celui des médias à utiliser ou de la quantité d'informations à diffuser. Les directives émises doivent de plus tenir compte des besoins en information des opérateurs dans la réalisation de leur tâche.

Cette définition instrumentale et normative a suscité de nombreuses études sur le choix des canaux à utiliser, sur les réseaux de communication et sur la façon dont ceux-ci sont utilisés pour véhiculer l'information. On s'est alors intéressé à diverses caractéristiques de ces réseaux comme leur configuration, leur accessibilité, leur densité (Putnam et Cheney, 1985). On a mis en relation les besoins informationnels de l'organisation, la richesse des médias et le design de l'organisation (Daft et Lengel, 1986). On a aussi identifié des rôles particuliers joués par certaines personnes à l'intérieur de ces réseaux comme la recherche, l'échange, la transmission et le filtrage des informations. On s'est donc interrogé sur l'utilisation des réseaux pour mieux cerner à qui, par qui et comment les messages devaient être envoyés.

En somme, on peut dire que la communication-productive, qui s'appuie sur une définition mécaniste de la communication et sur une conception hiérarchique de l'organisation, est de l'ordre $\mathrm{du}$ « faire faire ». Elle amène le gestionnaire à se poser des questions telles que : Quelles sont les informations utiles à l'accomplissement de la tâche? Quelles sont les connaissances préalables nécessaires à la compréhension du message ? Qui a besoin de ces informations et de ce savoir? Quel est le medium le plus adéquat pour transmettre ce message ? Ces questions visent à le sensibiliser à l'importance du facteur communication dans la réalisation des tâches à accomplir dans l'organisation, à évaluer non seulement l'efficience mais aussi l'efficacité des mécanismes de communication utilisés dans la production des biens, des services et de l'information.

\section{La communication intégratrice}

La communication intégratrice présente la communication comme une relation visant à rassembler les membres de la collectivité organisationnelle. La communication est alors comprise dans son sens premier $d$ »'unir ensemble » (cum unicare). Dans cette définition, la communication est décrite comme une interaction c'est-à-dire un dialogue, une relation entre sujets créateurs de sens. L'interaction comme action réciproque pose que les parties prenantes à la communication sont à la fois émetteurs et récepteurs. La communication-intégratrice permet ainsi à l'individu de se situer dans son environnement, de prendre part à l'action collective. Cette définition relationnelle de la communication repose sur une vision organiste (Sfez, 1991). Elle correspond en théorie de l'organisation (Morgan, 1989) à la représentation de l'organisation comme un organisme différencié en interaction avec son environnement ou comme une communauté culturelle.

Cette définition réintroduit l'individu comme sujet dans un système. Elle met l'accent sur le contexte qui donne du sens à cette relation. Elle décrit l'émetteur et le récepteur comme des partenaires dans l'acte de communication. La signification n'est plus seulement créée par l'émetteur, transmise dans le message et décodée par le récepteur mais elle est aussi créée par le récepteur. Le contenu du message n'est plus seulement opératoire ; il est aussi expressif. La communication comme comportement exprime des émotions, des sentiments, des attitudes. 

(Watzlawick et al.,1972) rejoint la conception de l'organisation comme une micro-société. Elle est en accord avec l'approche des relations humaines qui s'est développée de la fin des années 30 jusque dans les années 60 dans le climat pessimiste suivant la grande crise et la seconde guerre mondiale. Se posant en réaction au formalisme du modèle mécanique, cette nouvelle perspective a mis l'accent sur les relations informelles, la vie au sein des groupes de travail. Les chercheurs de l'école des relations humaines ont défini l'individu non plus seulement comme un « homo économicus ", mais aussi comme un être social qui est motivé par divers besoins dont celui d'affiliation à un groupe. Ils ont montré l'influence des normes de groupe sur la productivité des équipes de travail. Ils ont posé l'hypothèse que la performance de l'organisation était influencée par la satisfaction des travailleurs et ils ont prescrit le développement de mécanismes visant la participation des travailleurs (gestion par objectifs, groupes autonomes de travail etc). La révélation de l'influence de la structure informelle et des normes du groupe sur le comportement des individus, la recherche de la démocratie industrielle sont des traits caractéristiques de cette approche qui remettaient ainsi en question les visions traditionnelles du pouvoir, de la culture et de la structure de l'organisation.

(a communication comme moyen de mettre en relation des acteurs est aussi en concordance avec l'approche contingente de Lawrence et Lorsch (1973). Ces auteurs ont souligné que les organisations, pour s'adapter à leur environnement, créaient à l'interne des unités différenciées (production, marketing, finance etc.). Ils ont constaté, dans leur étude du phénomène de la différenciation, que ces unités avaient non seulement des préoccupations mais aussi des langages et des horizons temporels différents. Cette diversité, qui peut créer des clivages et amener la fragmentation de l'organisation, doit, selon eux, être contrebalancée par un effort d'intégration afin d'assurer la coordination des efforts de tous. Aussi ont-ils proposé la mise en place de groupes ou de fonctions d'intégration. Ils ont en outre affirmé que la capacité à gérer les conflits par une communication directe était gage d'une plus grande intégration. Ainsi, pour eux, la capacité à relier par la communication les groupes différenciés pour former une organisation intégrée est primordiale pour l'adaptation et la survie dans un environnement en changement.

Finalement, la communication comme outil relationnel a aussi des affinités avec l'approche de l'ingénierie culturelle qui s'est développée au cours des années 80 . Des ouvrages qui ont connu une grande popularité auprès des gestionnaires tels que In Search of Excellence (Peters et Waterman, 1982) et The Art of Japanese Management (Pascale et Athos, 1981) ont attiré l'attention sur l'influence de la culture sur la performance des organisations. Ces livres ont soutenu que la culture servait à cimenter l'organisation, que la présence de valeurs partagées créait une unité d'action et que la satisfaction des besoins d'affiliation suscitait la fidélité et l'engagement des membres de l'organisation. Ces études ont fait ressortir l'importance de certains éléments de communication tels que le langage, les logos, les rituels, les histoires et les symboles dans la constitution de la culture organisationnelle (Lemaître, 1985). Dans cette optique, la communication interne devient alors un moyen de relier les membres de l'organisation pour former un groupe plus homogène participant de la même culture. Cette littérature prescriptive prône la création de la culture par les leaders et sa diffusion dans l'organisation.

Ainsi, la communication relationnelle est orientée vers le développement et le maintien de la cohésion interne à l'intérieur des groupes, entre les unités et dans l'ensemble de 
l'organisation. La communication devient ainsi un instrument d'intégration. L'utilité de la communication repose alors dans sa capacité à générer la coopération au sein de l'organisation (Barnard, 1938), à faciliter l'assimilation des individus (Jablin, 1985), à harmoniser et à réduire les distances entre les groupes (Henriet et Boneu, 1990). La communication est alors sociabilité et socialisation. C'est pourquoi, dans cette approche, on valorise la communication informelle, orale, dans des échanges de face à face. La communication y est à la fois verticale et horizontale. Dans sa forme émergente, on peut l'observer, par exemple, dans les échanges lors de la pause café ou à la cafétéria ; dans sa forme planifiée, elle est visible dans les programmes d'intégration des nouveaux employés ou dans l'organisation de rencontres entre les groupes ou encore d'activités sociales.

La définition de la communication-intégratrice met l'emphase sur la création de liens. Aussi les chercheurs se sont-ils beaucoup intéressés à la relation supérieur-subordonné et à la notion de climat organisationnel. En fait, le postulat de base qui sous-tend cette approche est que l'amélioration du climat dans l'organisation entraînera une augmentation de la satisfaction au travail, provoquera un engagement accru et une productivité plus grande (Euske et Roberts, 1987). Dans cette approche, on fait l'hypothèse que la paix industrielle découlera automatiquement de la communication honnête entre gestionnaires et employés (Koehler et al., 1981). C'est pourquoi on recommande de développer la capacité d'écoute et une attitude d'ouverture et de confiance.

On propose aussi aux praticiens de créer un environnement communicationnel qui stimule les relations. On leur recommande alors d'informer l'individu sur sa place dans l'organisation, sur celle des autres personnes, sur l'évolution de l'entreprise au moyen de journaux internes, de communiqués ou de montages audio-visuels car on suppose que ces informations permettront de développer une connaissance mutuelle des personnes et des tâches (Gondrand, 1983) de même qu'un sentiment d'appartenance à la collectivité. On suggère aux gestionnaires d'initier aussi des occasions de rencontres et d'échanges (visites de dirigeants, fêtes etc.) afin de faciliter les contacts interpersonnels. Ces événements ont pour but de stimuler la convivialité, de favoriser l'interactivité et la solidarité (McQuail, 1987 ; Henriet et Boneu, 1990).

31 La communication intégratrice n'est toutefois pas exempte de problèmes. L'interconnection des réseaux informels facilite, pour le meilleur ou pour le pire, l'existence des rumeurs (Dupuy et al. 1988). Le développement d'une communauté de pensée peut engendrer le phénomène de "groupthink » c'est-à-dire l'homogénéisation des points de vue et la création de biais cognitifs communs. Le renforcement des relations entre individus peut entrainer des situations pathologiques comme la folie à deux et la double contrainte (Watzlawick et al. 1972). La communication comme relation n'est pas à l'abri de phénomènes tels que le malentendu, la manipulation et la collusion.

En résumé, on peut dire que la communication intégratrice, qui se base sur une définition comportementale de la communication et sur une vision collectiviste de l'organisation, est de l'ordre de l'« être ensemble ». Cette définition amène le gestionnaire à se poser diverses questions comme par exemple : les membres de l'organisation se connaissent-ils entre eux? Sont-ils capables de situer leur action dans le projet organisationnel et de comprendre les conséquences de leurs activités sur les autres participants? Sont-ils conscients des idées et des pratiques qui les rassemblent ou qui les divisent? Toutes ces questions ont pour but de le rendre plus conscient de la qualité des liens qui unissent les 
membres de l'organisation et de l'aider à mesurer le niveau d'intégration de la collectivité organisationnelle.

\section{La perspective interprétative}

La perspective interprétative s'intéresse aux points de vue des acteurs qui sont engagés dans la réalisation des activités sociales (Burrel et Morgan, 1979). Il s'agit donc d'une approche subjectiviste qui présente la société et l'organisation comme des créations humaines, produites à travers des processus émergents. Cette perspective met l'accent sur le quotidien et la performance des acteurs en situation. Contrairement à la perspective fonctionnaliste qui s'intéresse aux structures (macrosociologie), la perspective interprétative focalise sur les actions des individus (microsociologie). Pour les tenants de cette approche, l'ordre, le consensus, la cohésion ne sont pas donnés, ils sont créés au quotidien. La perspective interprétative bien qu'elle s'appuie sur la tradition de l'idéalisme allemand est relativement récente dans le champ de la gestion et de la communication.

\section{La communication « organisante »}

La communication « organisante » c'est la communication qui crée l'organisation. Dans cette perspective, on s'intéresse à ce que font les partenaires de la relation quand ils échangent des messages. La communication y est présentée comme une transaction par laquelle les partenaires bâtissent leur relation et leur identité, échangent de la valeur, construisent l'organisation. On ne peut plus, dès lors, parler vraiment de communication interne puisque l'organisation n'est plus un contenant dans lequel se produisent des activités de communication. Il s'agit plutôt de l'inverse : l'organisation est le produit des processus de communication (McDaniel Johnson, 1977). L'organisation est contenue dans la communication. Cette conception de la communication correspond à la définition de l'organisation comme un ensemble de processus qui la créent, la maintiennent et la défont (Weick, 1969).

Cette vision transactionnelle de la communication propose que l'échange de messages soit une activité créatrice. Selon Barnlund (dans Sfez, 1991 p.76) le sens n'est pas reçu, il est inventé c'est pourquoi il préfère utiliser le terme transaction plutôt que le terme interaction pour décrire la communication. Pour Barnlund (1971), les sujets ne reçoivent pas la signification, ils attribuent plutôt des significations aux différents objets de perception. Dans les relations interpersonnelles, afin de réduire l'ambiguïté, les acteurs assignent de façon expérimentale, en tenant compte du contexte spatial et temporel dans lequel ils se situent, des significations aux indices publics, privés, verbaux et non verbaux qu'ils observent. Chacun des partenaires tend alors à réguler les signaux qu'il émet, à progressivement reconnaitre les multiples significations que l'autre peut attacher à ses actions et à interpréter ses propres actions en fonction de la définition de l'autre. Ce processus d'ajustement mutuel et de vérification progressive du sens attribué tend à réduire l'incertitude de la situation. Ainsi, les partenaires ne font pas qu'émettre ou recevoir des messages: ils les interprètent, ils construisent à travers les séquences de messages une définition de leur réalité, ils confrontent leurs points de vue pour en arriver à une représentation commune (Putnam, 1983). En somme, ils construisent ensemble la signification. 
Dans la définition de la communication-intégratrice, la communication était décrite comme une interaction c'est-à-dire une action réciproque; dans la définition de la communication " organisante ", elle est plutôt décrite comme une transaction c'est-àdire une action conjointe qui vise à échanger et à créer de la valeur (Taylor, 1993), à parvenir à un accord à la suite de compromis ou d'accommodements, à créer des engagements. La définition transactionelle propose aussi une nouvelle conception des réseaux de communication. Dans cette optique, les comportements de communication n'ont pas lieu dans des réseaux. En fait, c'est plutôt par la communication que se constituent les réseaux (Hawes, 1974).

Cette vision de la communication est en correspondance avec une nouvelle conception de l'organisation qui ne la présente plus comme une entité mais comme un processus d'« organizing " (Weick, 1969). La structure de l'organisation est, dans cette nouvelle approche, le résultat d'un processus de structuration (Eraly, 1988), elle n'est plus une configuration mais une coactivation (Dow, 1988) ; l'organisation n'a plus une culture, elle est une culture (Smireich, 1983, Weick cité dans Kiechel, 1984), le pouvoir n'est plus une donnée fixe, il est produit dans la communication (Banks, 1990). Dans cette définition dynamique, c'est à travers les activités quotidiennes de ses membres que se construit l'organisation. Ce qui constituait des caractéristiques (culture, structure, système de pouvoir) de l'organisation devient alors le produit des processus collectifs de création de signification, de relations et d'identités.

La communication devient donc le processus de création de la collectivité organisationnelle et son utilité repose alors dans sa capacité à produire et à reproduire cette collectivité. Mais, pour que celle-ci survive à long terme, la communication doit aussi permettre le renouvellement de l'organisation. C'est pourquoi, la communication doit favoriser l'innovation. Cela suppose que dans la communication est inventée une nouvelle définition de l'organisation et sont construits de nouveaux réseaux. C'est pourquoi Gray et al. (1985) décrivent l'organisation comme un système de construction et de destruction de signification.

9 La communication " organisante » est collective, multidirectionnelle. Elle concerne tout autant la définition de la tâche que celle de la relation et de l'identité des acteurs (Taylor et Giroux, 1993). Elle est une conversation orale et écrite au cours de laquelle les membres tentent d'écrire le texte de l'organisation (Taylor, sous presse). Cette conversation est collective, ce qui ne veut pas dire qu'elle soit forcément égalitaire ; certains participants à cause de leur savoir, de leur autorité formelle et de leur ascendant peuvent avoir plus d'influence sur le processus de construction de l'organisation. Elle est multidirectionnelle mais non désordonnée; au fil du temps on peut observer des "patterns " dans les séquences d'interactions entre les participants (Krone et al., 1987) ainsi que des règles de communication qui sont construites et qui peuvent être plus ou moins comprises et appliquées par les acteurs.

La communication est donc à la fois le fondement de l'action organisée et l'outil de réflexion et de transformation de l'organisation. On la retrouve au quotidien dans toutes les activités de l'organisation et dans les échanges sur l'organisation. Dans sa forme émergente, elle est visible dans les discussions informelles sur les problèmes à résoudre ou sur le climat organisationnel. Elle ne peut être formellement planifiée puisqu'elle comporte malgré la présence de routines, une grande part d'improvisation (Hawes, 1973). Cependant le processus de conversation qui s'actualise au quotidien dans l'organisation peut être encadré, institutionnalisé dans la constitution de groupes de travail, dans la 
mise en place de cercles de qualité, dans l'instauration d'un mode de gestion participatif ou encore dans l'établissement d'un processus collectif de formulation stratégique (Giroux et Taylor, 1993).

41 La définition de la communication « organisante " met l'emphase sur les processus: les processus par lesquels se développe la structure et émergent de nouveaux réseaux (Monge et Eisenberg, 1987), les processus d'interprétation et de coorientation (Demers, 1990; McDaniel Johnson, 1977) qui permettent l'appropriation et l'actualisation de la statégie de l'organisation, les processus de création d'identité et de légitimité qui donnent un sens à l'action de l'individu et à l'action collective.

42 La définition de la communication "organisante » est relativement récente et n'a pas donné lieu encore à beaucoup de recherches de nature empirique opérationnalisant les concepts présentés. Toutefois on peut retrouver dans quelques études les manifestations de la communication « organisante ». Ainsi, par exemple, Roy (1960) relate comment les employés d'une manufacture structurent leur temps de travail et leurs relations, développent leur culture à travers la communication autour de certains thèmes. Pacanowsky (1988) décrit une nouvelle structure d'organisation : l'organisation en treillis. Dans ce type d'organisation où il n'y a pas d'organigramme, ce sont les membres qui au fur et à mesure de l'émergence de projets et de problèmes bâtissent les réseaux, inventent de nouvelles tâches et rassemblent les ressources pour les mener à bien. Boje (1991) montre comment les histoires sont, en contexte organisationnel, une performance collective, une forme de traitement de l'information et Gronn (1983) illustre comment, à travers les échanges langagiers, s'accomplit le contrôle dans une organisation scolaire.

L'approche de la communication « organisante » montre la mouvance de l'organisation qui est constamment en formation. Elle montre aussi que cette mouvance n'est pas un chaos. Elle est plutôt le reflet de la créativité dynamique des participants. Les problèmes que soulève cette approche ont trait à la participation à cette œuvre collective au fil du temps. Il convient alors de s'interroger sur les facteurs qui facilitent ou inhibent la conversation organisationnelle, sur les barrières et blocages qui peuvent limiter l'étendue de la participation au sein de la collectivité et sur les thèmes qui demeurent tabous. Une autre problématique pertinente que pose la perspective de la communication " organisante» est celle du déroulement de cette conversation dans le temps. Cette conversation peut être marquée de pauses, de longs silences ou encore de controverses et de chuchotements. Il serait donc intéressant de comprendre les différents modes de conversation et leur impact sur l'organisation.

En résumé, on peut dire que la définition de la communication "organisante », qui se fonde sur une définition transactionnelle de la communication et sur une description de l'organisation comme produit des activités de communication quotidiennes entre les acteurs, est de l'ordre du «faire ensemble». Cette définition processuelle pose au gestionnaire les questions suivantes: Qui a droit de parole? Quelle est l'étendue de la participation et sur quoi porte-t-elle? Comment favoriser l'expression et le partage des savoirs et des aspirations? Comment soutenir la continuité de cet effort collectif? Ces questions visent à évaluer la qualité de la conversation organisationnelle et le niveau de participation à cette œuvre collective. 


\section{La comparaison des trois définitions} communication productive son aspect mécanique, son occultation de la dimension humaine et l'aliénation que provoque le modèle classique d'organisation où la signification du travail est élaborée par ceux qui sont loin du terrain. On blâme la communication intégratrice pour son caractère manipulateur. En effet l'école des relations humaines tout comme l'approche de l'ingénierie culturelle valorisent les relations entre les membres de l'organisation via les normes du groupe ou les significations partagées mais seulement dans la mesure où celles-ci ne remettent pas en question le système de pouvoir en place et s'intègrent harmonieusement dans la vision des leaders. La satisfaction des besoins psychologiques et sociaux des travailleurs est alors perçue comme un autre outil de contrôle et d'amélioration du rendement. La communication «organisante» est, pour sa part, critiquée parce qu'elle déconstruit l'organisation précédemment réifiée. En replaçant l'organisation au niveau de la transaction, elle la fragmente faisant ainsi perdre de vue l'ensemble de la collectivité en action. De plus, cette définition de la communication interne offre encore peu de recommandations à ceux qui veulent influencer le déroulement du processus organisant. 
49 Cette critique de la communication "organisante» est en partie fondée. La communication « organisante » a jusqu'à présent fait l'objet de peu d'études empiriques. Son principal défi est de décrire comment elle produit, à travers des échanges éphémères, une collectivité régulée et stable. Il s'agit, comme le décrit Taylor (1993), de comprendre comment à travers les transactions qui ont lieu dans les conversations s'écrit et se transforme le texte organisationnel.

Pour cela la perspective de la communication « organisante » devra dépasser l'approche interprétative microsociologique dont elle est issue et s'alimenter aux courants de recherche actuels qui visent à résoudre la dualité entre le niveau micro et le niveau macro, entre l'action et la structure. Il faudra alors s'attacher à révéler la communication dans les chaînes d'interactions (Collins, 1987), les processus de traduction (Callon, 1985 ; Callon et Latour, 1981) de représentation (Knorr-Cetina, 1988) qui marquent la structuration de l'organisation (Giddens, 1984).

\section{Conclusion}

51 Dans ce texte, nous avons décrit et comparé trois définitions de la communication interne en faisant ressortir les conceptions de la communication et de l'organisation sur lesquelles elles reposent ainsi que les buts qu'elles assignent et les problématiques qu'elles soulèvent. Ces définitions se sont succédées dans le temps. La définition de la communication intégratrice a été développée en réaction aux limites de la communication productive ; celle de la communication " organisante » en réaction à la réification de l'organisation inscrite dans les deux précédentes. La communication « organisante » nous apparaît être la plus proche de la réalité organisationnelle vécue au quotidien. Elle est cependant plus complexe et nécessite un ancrage dans des études empiriques. Malgré ses lacunes elle n'en constitue pas moins un champ d'investigation prometteur. 
Tableau 1. Définitions de la communication interne

\begin{tabular}{|c|c|c|c|}
\hline & \multicolumn{2}{|c|}{ Fonctionnaliste } & \multirow{2}{*}{\begin{tabular}{|l} 
Interprétative \\
Faire ensemble
\end{tabular}} \\
\hline & Faire faire & Etre ensemble & \\
\hline Définitions & Productive & Intégratrice & "Organisante" \\
\hline $\begin{array}{l}\text { Définitions de } \\
\text { l'organisation }\end{array}$ & Machine & Collectivité & $\begin{array}{l}\text { Construction } \\
\text { sociale }\end{array}$ \\
\hline $\begin{array}{c}\text { Conceptions de } \\
\text { Structure }\end{array}$ & Configuration & $\begin{array}{l}\text { Formelle et } \\
\text { informelle }\end{array}$ & Coactivation \\
\hline Culture & $\begin{array}{l}\text { Valeurs de la } \\
\text { société }\end{array}$ & $\begin{array}{c}\text { Valeurs } \\
\text { partagées }\end{array}$ & Performance \\
\hline Pouvoir & Délégué & $\begin{array}{l}\text { Formel et } \\
\text { informel }\end{array}$ & Performance \\
\hline $\begin{array}{l}\text { Définitions de } \\
\text { la communica- } \\
\text { tion }\end{array}$ & Transmission & Interaction & Transaction \\
\hline $\begin{array}{l}\text { Buts de la com- } \\
\text { munication }\end{array}$ & $\begin{array}{l}\text { Réalisation de la } \\
\text { tâche }\end{array}$ & Cohésion & $\begin{array}{l}\text { Maintien } \\
\text { innovation }\end{array}$ \\
\hline $\begin{array}{c}\text { Caractéristiques } \\
\text { de la communi- } \\
\text { cation }\end{array}$ & $\begin{array}{l}\text { Formelle } \\
\text { Verticale }\end{array}$ & $\begin{array}{l}\text { Formelle et } \\
\text { informelle } \\
\text { verticale et } \\
\text { horizontale }\end{array}$ & Collective \\
\hline $\begin{array}{c}\text { Manifestations } \\
\text { spontanées } \\
\text { planifiées }\end{array}$ & $\begin{array}{l}\text { Directives } \\
\text { Manuels }\end{array}$ & $\begin{array}{l}\text { Conversations } \\
\text { Réunions } \\
\text { sociales }\end{array}$ & $\begin{array}{l}\text { Discussions } \\
\text { Groupe de } \\
\text { travail }\end{array}$ \\
\hline Focus & $\begin{array}{l}\text { Messages } \\
\text { Canaux } \\
\text { Réseaux }\end{array}$ & $\begin{array}{l}\text { Relations et } \\
\text { climat }\end{array}$ & $\begin{array}{l}\text { Processus de } \\
\text { structuration } \\
\text { coorientation } \\
\text { légitimation }\end{array}$ \\
\hline Problématique & $\begin{array}{l}\text { Qualité du mes- } \\
\text { sage et de sa } \\
\text { transmission }\end{array}$ & $\begin{array}{l}\text { Rumeurs } \\
\text { "Groupthink" }\end{array}$ & $\begin{array}{l}\text { Etendue et conti- } \\
\text { nuité de la parti- } \\
\text { cipation }\end{array}$ \\
\hline
\end{tabular}

\section{BIBLIOGRAPHIE}

Aktouf, Omar (1989a), Le management entre tradition et renouvellement, Boucherville, Gaétan Morin.

Aktouf, Omar (1989b), " Parole, travail et productivité », Actes du colloque Travail et pratiques langagières, CRG-Ecole Polytechnique, Paris 24-26 avril.

Auvinet, Jean-Marie, Boyer, Luc, Bureau Romain, Pierre Chappaz et Guillaume de Vulpian (1990), La communication interne au cœur du management, Paris, Édition d'organisation.

Axley, Stephen R.(1984), Managerial and Organizational Communication in Terms of the Conduit Metaphor, Academy of Management Review, vol. 9, n 3, p. 428-437.

Banks, Stephen (1990), Organizational Power as Communication Praxis dans Corman, Steven R., Banks, Stephen P. Bantz Charles R., et Michael E. Mayer (eds), Foundations of Organizational Communication, New-York, Longman, p. 275-281.

Barnard, Chester (1938), The Function of the Executive, Cambridge, Harvard University Press.

Barnlund, Dean, C.(1971), A Transactional Model of Communication, dans Barker, Larry et Kibler Robert (eds) Speech Communication Behavior, Definitions and Principles, Englewood Cliffs, Prentice Hall, pp. 71-81. 
Bartoli, Annie (1990), Communication et Organisation pour une politique générale cohérente, Paris, Editions d'organisation, Collection campus, entreprise, université.

Boje, David M.(1991), « The Storytelling Organization : A Study of Story Preformance in an OfficeSupply Firm », Administrative Science Quarterly, vol. 36, 1991, p. 106-126.

Burrell, Gibson et Gareth Morgan (1979), Sociological Paradigms and Organisational Analysis, London, Heinemann.

Callon Michel (1985), Some Elements of a Sociology of Translation: Domestication of the Scallops and the Fishermen of St Brieuc Bay dans J. Law (éd.) Power, Action and Belief : A New Sociology of Knowledge, London Routledge et Keagan Paul, p. 196-234.

Callon Michel et Bruno Latour (1981), Unscrewing the Big Leviathan, or How Actors Macrostructure Reality and How Sociologists Help them do so, dans Knorr-Cetina Karin et Aaron Cicourel (éds), Advances in Social Theories and Methodology : Toward an Intégration of Micro and Macro Sociologies, London, Routledge et Keagan Paul, p. 277-303.

Collins, Randall (1987), Interaction Ritual Chains, Power and Property: The Micro-Macro Connections as an Empirically Based Theoretical Problem dans Alexander Jeffrey, C. Bernhard, Giesen Richard (éds) The Micro-Macro Link, University of California Press, p. 193-201.

Daft, Richard L. et Robert H. Lengel (1986), Organizational Information Requirements, Media Richness and Structural Design, Management Science, vol. 32, n 5, p. 554-571.

Demers, Christiane (1991), Le changement radical vu de l'intérieur : la diffusion stratégique dans les organisations complexes, Gestion, mai, p. 22-31.

Dow, Gregory, K.(1988), Configurational and Coactivational Views of Organizational Structure, Academy of Management Review, vol. 13, no ${ }^{\circ}$ 1, p. 53-64.

Dupuy, Emmanuel, Devers Thomas et Isabelle Raynaud (1988), La communication interne, vers l'entreprise transparente, Paris, Les Editions d'organisation.

Eisenberg, Eric (1984), « Ambiguity as a Strategy in Organizational Communication », Communication Monographs, vol. 5, septembre, p. 227-242.

Euske, Nancy A.., Roberts, Karlene (1987), Evolving Definition in Organization Theory : Communication Implications, dans Jablin, F., Putnam L., Roberts K, Porter L. (eds) Handbook of Organizational Communication, Beverly Hills, Sage, p. 41-70.

Eraly, Alain (1988), La structuration de l'organisation, Bruxelles, Editions de l'Université de Bruxelles.

Farace, Richard V., Monge, Peter R. et HAMISH M. Russel (1977), Communicating and Organizing, Don Mills, Addison -Wesley.

Feldman M.S.et James G. March, Information in Organizations as Signal and Symbol Administrative Science Quarterly, vol. 26, 1981, p. 171-186.

Giddens, Anthony (1984), The Constitution of Society, Oxford, Polity Press.

Giroux, Nicole et James TAYLOR, Le changement par la conversation stratégique » Montréal document de travail Giscor, 1993

Gondrand, François (1983), L'information dans les entreprises et les organisations, Paris, Éditions d'organisation.

Gray B., Bougon, M. et Donnellon A.(1985), Organizations as Construction and Destruction of Meaning, Journal of Management Studies, vol. 1, nº 2, p. 83-98. 
Gronn, Peter, C.(1983), Talk as the Work: The Accomplishment of School Administration, Administrative Science Quarterly, vol. 28, 1983, p. 1-21.

Hawes Leonard C.(1974), Social Collectivities As Communication, Quarterly Journal of Speech, vol. 60, p. 497-502.

Hawes, Leonard C.(1973), Interpersonal Communication : The Enactment of Routines dans J. M Makay et Charles Merril (éds) Explorations in Speech Communication, Columbus Ohio, p. 71-91.

Henriet, Bruno, Boneu, François (1990), Audit de la communication interne, Paris Editions d'organisation.

Jablin, Fredric M.(1985), Task/Work Relationships : A Life-Span Definition, dans Knapp, Mark L., Miller Gerald R. (eds) Handbook of Interpersonnal Communication, Beverly Hills, Sage, p. 615-655.

Kiechel, W III (1984), Sniping at Strategic Planning (Interview with Himself), Planning Review, May, p. 11.

Knorr-Cetina, Karin (1988), The Micro-social Order, Towards a Reconception dans Nigel G. Fielding, Actions and Structure, Beverly Hills, Sage, p. 20-54.

Koehler, Jerry W., Anatol, Karl W. E., et Ronald L. APPLEBAUM (1981), Organizational Communication, Behavioral Définitions, Toronto, Holt, Rinehart and Winston.

Krone, Kathleen, Jablin, Frederick et Linda Putnam (1987), Communication Theory and Organizational Communication : Multiple Définitions, dans Jablin, F., Putnam L., Roberts K, Porter L. (eds) Handbook of Organizational Communication, Beverly Hills, Sage, p. 18-41.

Laramée, Alain (1989), La communication dans les organisations, Presses de l'Université du Québec, Sillery.

Lawrence, P.R., Lorsh, J.W.(1973), Adapter les structures de l'entreprise : intégratrice ou différenciation, Paris, Édition d'organisation,.

Lemaitre, Nadine (1985), La culture d'entreprise, facteur de performance Gestion, février, p. 19-25.

March, James et Herbert Simon (1958), Organizations, New-York, Wiley.

Mcdaniel Johnson, Bonnie (1977), Communication The Process of Organizing, Toronto, Allyn and Bacon.

Mcquail, Denis (1987), Functions of Communication : a Non functionalist Overview dans Berger, Charles R., Chaffee, Steven H. (eds), Handbook of Communication Science, Newbury Park, Sage, p. 327-350.

Mintzberg, Henry (1979), The Structuring of Organizations, Englewood Cliffs, N.J., Prentice Hall. Morgan, Gareth (1989), Images de l'organisation, Québec, Presses de l'Université Laval.

Monge Peter, R., Eisenberg, Eric M.Q987), Emergent Communication Networks dans Jablin, F., Putnam L., Roberts K, Porter L. (eds) Handbook of Organizational Communication, Beverly Hills, Sage, 1987, p. 304-343.

Kmichael (1988), Communication in the Empowering Organization ", Communication Yearbook, vol 11, p. 356-379.

Pascale, R.T., Athos, A.G.(19810, The Art of Japanese Management, New-York, Simon \& Shusster/ Warner Books

Peters, Thomas J., Waterman Robert H.Q982), In Search of Excellence, New-York, Warner Books. 
Putnam, Linda L.(1982), Paradigms for Organizational Communication Reserch : An Overview and Synthesis, The Western Journal of Speech Communication, vol. 46, spring, p. 192-206.

Putnam, Linda L.(1983), The Interprétative Définition An Alternative to Functionalism dans Putnam, L., et M. Pacanowsky (eds) Communication And Organizations An Interpretative Approach, Beverly

Hills, Sage, p. 31-55.

Putnam, Linda L., Cheney, George (1990), Organizational Communication : Historical Development And Future Directions, dans Corman, Steven R., Banks, Stephen P. Bantz Charles R., et Michael E. Mayer (eds), Foundations of Organizational Communication, New-York, Longman, p. 169-171.

Rogers Everett, Agarwala-Rogers, REHKA (1980), Three Schools of Organizational Behavior, dans Ferguson Stewart, Sherry Devereaux Ferguson (eds) Intercom : Readings in Organizational Communication, Rochelle Park, Hayden, p. 2-33.

Redding, Charles, W.(1985), Stumbling Toward Identity The Emergence of Organizational communication as a Field of Study, McPhee, Robert D. et Philip K Tompkins (éds) Organizational Communication : Traditional Themes and New Directions, Newbury Park, Sage annual Review of Communication Research, vol. 13, p. 15-55.

Roy, Donald F.(1959-1960), « Banana, Time : Job Satisfaction and Informal Interaction », Human Organization, vol. 18, winter, p. 156-168.

Schwebig, Philippe (1988), Les communications de l'entreprise, au-delà de l'image, Montréal, McGrawHill, Coll. Stratégie et Management.

Sfez, Lucien (1991), La communication, Paris, Presses Universitaires de France, coll. Que sais-je?

Simon, Herbert, A. (1990), The Fine Art of Issuing Orders dans Corman, Steven R., Banks, Stephen P. Bantz Charles R., et Michael E. Mayer (eds), Foundations of Organizational Communication, NewYork, Longman, p. 169-171.

Smircich, Linda (1983), Studying Organisations as Cultures, dans Beyond Method, Strategies for Social Research, Beverly Hills, Sage, p. 160-173.

Taylor, James, Rethinking the Theory of Organization, Ablex, sous presse.

Taylor, James (1993), La dynamique de changement organisationnel, une théorie conversation/texte de la communication et ses implications, Communication et organisation, $\mathrm{n}^{\circ} 3$, mai.

Taylor James et Nicole GIROUX (1993), The Rhetoric of Management Conversation and Text, présentation à la conférence d'EGOS Paris, juillet.

Watzlawick, P., Helmick-Beavin J. et D. Jackson (1972), Une logique de la Communication, Paris Éditions du Seuil.

Weick, Karl, E.(1969), The Social Psychology of Organizing, Don Mills, Addison Westley.

\section{RÉSUMÉS}

Cet article présente trois définitions de la communication interne qui sont issues des perspectives fonctionnaliste et interprétative : la communication productive, la communication intégratrice et la communication « organisante ». Il montre comment ces définitions s'appuient sur des conceptions de l'organisation et de la communication qui sont sensiblement différentes. Il soulève les problématiques privilégiées par chacune de ces définitions et signale les avenues de recherche qu'ouvre la définition de la communication interne comme processus d'organisation. 
This article presents three definitions of internal communication stemming from the functionalist and interpretative paradigms: productive, integrative and organizing communication. These definitions illustrate different conceptions of organization and communication and draw attention to different problems of communication in organizations. This paper proposes to put more emphasis in research on communication as an organizing process.

\section{AUTEUR}

\section{NICOLE GIROUX}

Nicole Giroux est professeur de communication des organisations à l'Université de Montréal et membre du Groupe d'intervention stratégique en communication organisationnelle (GISCOR). Elle a aussi enseigné la gestion, la stratégie et le design des organisations à l'École des Hautes Etudes Commerciales de Montréal et à l'Université du Québec à Montréal. Dans ses recherches, elle s'intéresse à la communication dans le processus stratégique (innovation, collaboration, fusion-acquisition, désinvestissement) et dans le processus de changement organisationnel plus particulièrement dans les institutions et les organisations participatives. 